\title{
Entanglement Entropy and Mutual Information in Bose-Einstein Condensates
}

\author{
Wenxin Ding and Kun Yang \\ NHMFL and Department of Physics, Florida State University, Tallahassee, Florida 32306, USA
}

(Dated: August 24, 2018)

\begin{abstract}
In this paper we study the entanglement properties of free non-relativistic Bose gases. At zero temperature, we calculate the bipartite block entanglement entropy of the system, and find that it diverges logarithmically with the particle number in the subsystem. For finite temperatures, we study the mutual information between the two blocks. We first analytically study an infiniterange hopping model, then numerically study a set of long-range hopping models in one-dimension that exhibit Bose-Einstein condensation. In both cases we find that a Bose-Einstein condensate, if present, makes a divergent contribution to the mutual information which is proportional to the logarithm of the number of particles in the condensate in the subsystem. The prefactor of the logarithmic divergent term is model dependent.

PACS numbers: 03.75.Gg
\end{abstract}

\section{INTRODUCTION}

Entanglement, as measured by, e.g., bipartite block entanglement entropy, is playing an increasingly important role in the study of condensed-matter or quantum many-body physics, both conceptually and quantitatively. It has been used as a very useful and in some cases indispensable way to characterize phases and phase transitions, especially for phases and quantum phase transitions in strongly correlated fermionic or spin systems (for a review, see Ref. [1]). For bosonic systems, studies of entanglement entropy have mostly focused on relativistic free bosonic field theories [2-4], which are equivalent to coupled harmonic oscillator systems (for reviews, see Refs. [5] and [6]).

In this paper we study the entanglement properties of free non-relativistic Bose gases. In addition to interest in its own right, our motivation also comes in part from the following consideration. In recent studies it has been shown that entanglement is enhanced at quantum critical points [7] and strongly correlated phases with topological order [8], in the form of either violation of area law [2,3,9-20], or subleading corrections to the area law that diverges with block size [21-23] (usually in a logarithmic fashion). On the other hand there have been relatively few studies of the behavior of entanglement entropy in states with traditional long-range order [24-26]. In a recent work [27], we calculated the block entanglement entropy of some exactly soluble spin models that exhibit ferromagnetic or antiferromagnetic long-range order in the ground state, and found that such conventional orders also lead to logarithmically divergent contribution to the entropy. Bose-Einstein condensation (BEC) is perhaps the simplest example of conventional ordering. It is thus natural to study its entanglement properties. As we are going to show, a Bose-Einstein condensate (referred to as a condensate from now on) indeed makes a logarithmically divergent contribution to the entropy as well.

Besides the entanglement entropy of the ground state, the entanglement properties of system at finite temperature are also of great interest. However, the entanglement entropy is only well-defined for a pure state. For a system that is described by a mixed density matrix, the von Neumann entropy of the reduced density matrix becomes different for the two parts of the bipartite systems. In such cases, there is a natural extension of the entanglement entropy that one can work with - the mutual information $[28,29]$. We will show that a condensate, when present, makes a logarithmically divergent contribution to the mutual information.

This paper is organized as follows. In Sec. II we study the ground state entanglement entropy of a generic free boson model that is translationally invariant [30]. In Sec. III we introduce an infinite-range hopping model for bosons which is exactly solvable, and calculate the mutual information analytically. In Sec. IV we introduce a long-range hopping model for bosons in one-dimension (1D) which exhibits a finite temperature BEC for a certain parameter range, then we present a numerical study of the mutual information for this model. In the end, we summarize and discuss the results of this paper in Sec. V.

\section{ZERO TEMPERATURE: ENTANGLEMENT ENTROPY OF FREE BOSONS}

Consider a general Hamiltonian of free bosons hopping on a lattice of size $L$ :

$$
H=-\sum_{i j} t_{i j} \hat{a}_{i}^{\dagger} \hat{a}_{j},
$$


where $t_{i j}>0, \hat{a}_{i}\left(\hat{a}_{i}^{\dagger}\right)$ 's are the bosonic annihilation (creation) operators. If the system is translationally invariant, $t_{i j}=t_{i-j}$, then the Hamiltonian can be diagonalized by Fourier transformation:

$$
H=\sum_{k} \varepsilon(k) \hat{b}_{k}^{\dagger} \hat{b}_{k}
$$

where $\hat{b}_{k}=\frac{1}{\sqrt{L}} \sum_{j} e^{-i j k} \hat{a}_{j}$ is the annihilation operator in $k$ space. In most generic cases, the ground state is the $k=0$ state. At zero temperature, all particles fall into the ground state. For a system containing $N$ particles, the ground state is given by:

$$
\left|\Psi_{0}\right\rangle=\frac{1}{\sqrt{N !}}\left(\hat{b}_{0}^{\dagger}\right)^{N}|0\rangle=\frac{1}{\sqrt{N !}}\left(\frac{1}{\sqrt{L}} \sum_{j} \hat{a}_{j}^{\dagger}\right)^{N}|0\rangle
$$

To consider its bipartite block entanglement entropy, we divide the system of size $L$ in two parts, and label them $A$ and $B$ respectively. Let the sizes of each part be $L_{A}$ and $L_{B}, L_{A}+L_{B}=L$, and define

$$
\hat{a}_{A}^{\dagger}=\frac{1}{\sqrt{L_{A}}} \sum_{j \in A} \hat{a}_{j}^{\dagger}, \hat{a}_{B}^{\dagger}=\frac{1}{\sqrt{L_{B}}} \sum_{j \in B} \hat{a}_{j}^{\dagger}
$$

Then we can write $\left|\Psi_{0}\right\rangle$ as:

$$
\begin{aligned}
\left|\Psi_{0}\right\rangle & =\frac{L^{-N / 2}}{\sqrt{N !}}\left(\sqrt{L_{A}} \hat{a}_{A}+\sqrt{L_{B}} \hat{a}_{B}\right)^{N}|0\rangle=\frac{L^{-N / 2}}{\sqrt{N !}} \sum_{l=0}^{N} \frac{N !}{(N-l) ! l !}\left(\sqrt{L_{A}} \hat{a}_{A}^{\dagger}\right)^{l}\left(\sqrt{L_{B}} \hat{a}_{B}^{\dagger}\right)^{N-l} \\
& =L^{-N / 2} \sum_{l} \sqrt{\frac{N !}{(N-l) ! l !}} L_{A}^{l / 2} L_{B}^{(N-l) / 2}\left[\frac{1}{\sqrt{l !}} \hat{a}_{A}^{\dagger l} \frac{1}{\sqrt{(N-l) !}} \hat{a}_{B}^{\dagger N-l}|0\rangle\right] \\
& =\sum_{l} \sqrt{\lambda_{l}}|l\rangle_{A} \otimes|N-l\rangle_{B}
\end{aligned}
$$

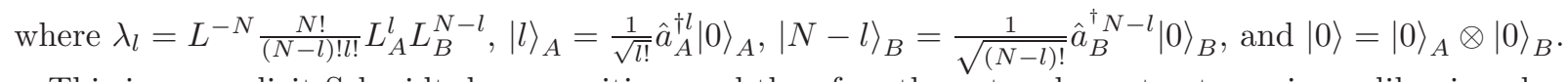

This is an explicit Schmidt decomposition, and therefore the entanglement entropy is readily given by:

$$
E=-\sum_{l} \lambda_{l} \ln \lambda_{l}
$$

We are interested in the asymptotic behavior in two limiting cases: (1) the equal partition case; (2) size of $B$ is substantially larger than $A$, i.e., $L_{B} \gg L_{A}$.

(i) Equal partition, $L_{A}=L_{B}=\frac{L}{2}$ :

$$
\lambda_{l}=\frac{N !}{l !(N-l) !} \frac{L_{A}^{l} L_{B}^{N-l}}{L^{N}}=\frac{N !}{l !(N-l) ! 2^{N}}=\frac{N !}{\left(\frac{N}{2}\right) !} \frac{\left(\frac{N}{2}\right) ! 2^{N}}{\left(\frac{N}{2}-\left(\frac{N}{2}-l\right)\right) !\left(\frac{N}{2}+\left(\frac{N}{2}-l\right)\right) !} .
$$

Let $x=\frac{N}{2}-l$, then $x \in\left[-\frac{N}{2}, \frac{N}{2}\right]$, and we can denote $\lambda_{l}$ as $\lambda_{x}=\frac{2^{N} N !}{\left(\frac{N}{2}\right) !} \frac{\left(\frac{N}{2}\right) !}{\left(\frac{N}{2}-x\right) !\left(\frac{N}{2}+x\right) !}$ which can be approximated by a Gaussian distribution factor $\lambda_{x} \sim e^{\frac{-2 x^{2}}{N}}$ when $N$ is large. In the limit $N \rightarrow \infty$, the summation over $n$ (or $x$ ) can be approximated by an integral. Also in this limit, the Gaussian factor is sharply peaked around $x=0$, the integral region can be extended to from minus infinity to infinity. Using the fact that $\sum_{x} \lambda_{x} \simeq \int_{-\infty}^{\infty} \lambda(x) d x=1$, we arrive at

$$
\lambda(x) \simeq \sqrt{\frac{2}{N \pi}} e^{-\frac{2 x^{2}}{N}}
$$

The entanglement entropy is then

$$
E \simeq-\int_{-\infty}^{\infty} \lambda(x) \ln \lambda(x) d x=\frac{1}{2}\left(1+\ln \left(\frac{N \pi}{2}\right)\right)=\frac{1}{2} \ln N+\mathcal{O}(1)
$$

(ii) Unequal partition, $L_{B} \gg L_{A}$ : 
If $L_{B} \gg L_{A}, L \rightarrow \infty$, but keep $\frac{N}{L} \rightarrow\langle n\rangle$ (fixed), the distribution of $\lambda_{l}$ approaches a Poisson distribution:

$$
\lambda_{l}=\frac{N !}{l !(N-l) !} \frac{L_{A}^{l} L_{B}^{N-l}}{L^{N}} \stackrel{N \rightarrow \infty}{\longrightarrow} \frac{\left(L_{A}\langle n\rangle\right)^{l} e^{-L_{A}\langle n\rangle}}{l !} .
$$

The entropy of the Poisson distribution, which in this case is our entanglement entropy, is known to be:

$$
\begin{aligned}
E & =\frac{1}{2}\left[1+\ln \left(2 \pi L_{A}\langle n\rangle\right)\right]-\frac{1}{12 L_{A}\langle n\rangle}+O\left(\frac{1}{\left(L_{A}\langle n\rangle\right)^{2}}\right) \\
& =\frac{1}{2}\left[1+\ln \left(2 \pi N_{A}\right)\right]-\frac{1}{12 N_{A}}+O\left(\frac{1}{\left(N_{A}\right)^{2}}\right)=\frac{1}{2} \ln N_{A}+\mathcal{O}(1),
\end{aligned}
$$

where $N_{A}=L_{A}\langle n\rangle$ is the average particle number in subsystem $A$.

Therefore, we find, in both cases, that the leading term of the mutual information goes as $\frac{1}{2} \ln N_{A}$ for $L_{A} \leq L_{B}$.

\section{MUTUAL INFORMATION: ANALYTIC STUDY OF AN INFINITE-RANGE HOPPING MODEL}

In this section, we will study the natural generalization of entanglement entropy at finite temperature: the mutual information, which is defined as

$$
E_{M}=\frac{1}{2}\left(E_{A}+E_{B}-S\right)
$$

where $E_{A}$ and $E_{B}$ are the von Neumann entropy of the reduced density matrices of subsystems A and B, respectively, and $S$ is the entropy of the whole system. Note that at finite temperature $E_{A}$ and $E_{B}$ are no longer the same due to the fact that the system is described by a mixed density matrix. We must emphasize here that our definition of mutual information differs from its usual definition [31] by a factor of 2 so that it will converge to the entanglement entropy when the system approaches a pure state.

\subsection{Model, spectrum and thermodynamic properties}

In order to facilitate an exact solution, we consider the following infinite-range hopping model which is obtained by setting $t_{i j}$ in Eq. (1) to a constant properly scaled by the system size $t_{i j}=t / L$ so that the thermodynamic limit is well-defined. The Hamiltonian is then

$$
H=-\frac{t}{L} \sum_{i, j} \hat{a}_{i}^{\dagger} \hat{a}_{j}=-\frac{t}{L}\left(\sum_{i} \hat{a}_{i}^{\dagger}\right)\left(\sum_{j} \hat{a}_{j}\right) .
$$

By substituting the Fourier transform of $\hat{a}_{j}$ 's defined in Sec. II, $\hat{b}_{k}=\frac{1}{\sqrt{L}} \sum_{j} e^{-i j k} \hat{a}_{j}$, one obtains:

$$
H=-t \hat{b}_{0}^{\dagger} \hat{b}_{0}
$$

This model has a very simple spectrum with a ground state with energy $-t$, and all the other excited states are degenerate with zero energy. This particularly simplified spectrum makes an exact solution possible.

To study the finite temperature properties of this model, we will work with the grand canonical ensemble (GCE), in which the chemical potential $\mu$ is introduced to control the average density of the system. This model exhibits BEC at finite temperature $T_{C}$. To determine $T_{C}$, we start by considering a system of finite size $L$, and its occupation numbers are:

$$
\left\langle N_{k=0}\right\rangle=\left\langle N_{0}\right\rangle=\frac{1}{e^{\beta(-t-\mu)}-1},\left\langle N_{k}\right\rangle=\frac{1}{e^{\beta(-\mu)}-1} \text { for } k \neq 0 .
$$

Here $\left\langle N_{0}\right\rangle$ and $\left\langle N_{k}\right\rangle$ denote average occupation numbers for the corresponding states in $k$-space; $\beta=\frac{1}{T}$ is the inverse temperature. From this point on, when we write $\left\langle N_{k}\right\rangle$, it immediately indicates $k \neq 0$. The average total particle number of the system will be denoted as $\langle N\rangle$. To identify $T_{C}$, we know in the thermodynamic limit, when $T \rightarrow T_{C}+0^{+}$, 
$\mu \rightarrow E_{k=0}=-t$, and $\frac{\left\langle N_{0}\right\rangle}{N} \rightarrow 0$. Therefore, $\left\langle N_{k}\right\rangle=\frac{1}{e^{\beta_{c} t}-1} \simeq \frac{\langle N\rangle}{L}=\langle n\rangle$ where $\langle n\rangle$ is the average particle density. So we obtain:

$$
T_{C}=\frac{t}{\ln (1+1 /\langle n\rangle)}
$$

Above $T_{C},\langle n\rangle=\frac{L-1}{L} \frac{1}{e^{-\beta \mu}-1}+\frac{1}{L} \frac{1}{e^{\beta(-t-\mu)}-1}$, from which in the large $L$ limit we can derive that

$$
\mu=-T \ln \left(1+\frac{1}{\langle n\rangle}\right) .
$$

$\mu$ has a finite size correction which is negligible above $T_{C}$, but will become important below $T_{C}$.

We also know that the partition function of the system in GCE. bears the following form:

$$
Z=\left(\frac{1}{1-e^{\beta \mu}}\right)^{L-1} \frac{1}{1-e^{\beta(t+\mu)}},
$$

from which it is easy to show that the entropy in GCE. takes the following form:

$$
\begin{aligned}
S & =-\frac{\partial \Omega}{\partial T}=\ln Z-\frac{1}{T} \frac{\partial}{\partial \beta} \ln Z \\
& =\left(1+\left\langle N_{0}\right\rangle\right) \ln \left(1+\left\langle N_{0}\right\rangle\right)-\left\langle N_{0}\right\rangle \ln N_{0}+(L-1)\left[\left(1+\left\langle N_{k}\right\rangle\right) \ln \left(1+\left\langle N_{k}\right\rangle\right)-\left\langle N_{k}\right\rangle \ln \left\langle N_{k}\right\rangle\right] .
\end{aligned}
$$

Anticipating later relevance, we are particularly interested in the behavior of finite size systems near $T_{C}$. For a finite system, the chemical potential $\mu$ is no longer strictly equal the ground state energy below $T_{C}$, but picks up a finite size correction $\delta \mu$ determined by the following condition:

$$
\frac{1}{e^{-\beta \delta \mu}-1}=\left\langle N_{0}\right\rangle,
$$

from which we can easily derive that

$$
\delta \mu=-T \ln \left(1+\frac{1}{\left\langle N_{0}\right\rangle}\right)
$$

Consider $T=T_{C}=\frac{t}{\ln (1+1 /\langle n\rangle)}$, and make use of the following fact

$$
\left\langle N_{0}\right\rangle=\langle N\rangle-\sum_{k \neq 0}\left\langle N_{k}\right\rangle=\langle N\rangle-\frac{L-1}{e^{\beta(t-\delta \mu)}-1},
$$

we obtain

$$
\left\langle N_{0}\right\rangle=\langle N\rangle-\frac{L-1}{e^{\beta_{c}(t-\delta \mu)}-1}=\langle N\rangle-\frac{L-1}{(1+L /\langle N\rangle)\left(1+1 /\left\langle N_{0}\right\rangle\right)-1} .
$$

This equation can be solved to give $\left\langle N_{0}\right\rangle$ as a function of system size $L$ at a given density $\langle n\rangle=\langle N\rangle / L$, at $T=T_{C}$ :

$$
\left\langle N_{0}\right\rangle=\sqrt{L} \sqrt{\left(\frac{\langle N\rangle}{L}\right)^{2}+\frac{\langle N\rangle}{L}+\frac{1}{4 L}} \simeq \sqrt{L} \sqrt{\langle n\rangle^{2}+\langle n\rangle} .
$$

Even though this divergent $N_{0}$ does not affect the thermodynamic behavior of the system, as we will see later it makes a (leading) divergent contribution to the mutual information at $T=T_{C}$ depending on how the system is partitioned, or specifically how large is the subsystem size $L_{A}$ compared with this $\sqrt{L}$ divergence.

\subsection{Formalism and issues}

In the following part, we will use Peschel's result [32] on the reduced density matrix of a Gaussian state:

$$
\rho_{A}=\mathcal{K} e^{\left\{\ln \left((1+G) G^{-1}\right)\right\}_{i j}^{T} \hat{a}_{i}^{\dagger} \hat{a}_{j}}
$$


where $G_{i j}=\left\langle\hat{a}_{i}^{\dagger} \hat{a}_{j}\right\rangle$ is the two point correlation function matrix truncated within the subsystem, and $\mathcal{K}$ is the normalization factor. The entropy is given as

$$
E_{A}=\sum_{l}\left[\left(1+g_{l}\right) \ln \left(1+g_{l}\right)-g_{l} \ln g_{l}\right],
$$

where $g_{l}$ 's are the complete set of eigenvalues of $G$ 's (after truncation). Actually this formula also applies to the original system.

We must note that, this formula does not lead to the correct zero temperature limit for the entropy. At zero temperature, $G_{i j}=\left\langle a_{i}^{\dagger} a_{j}\right\rangle=\langle n\rangle$. Its eigenvalues are all zero except for one: $g_{0}=\langle n\rangle L=\langle N\rangle$, which gives us a non-zero entropy $S_{T=0}=(\langle N\rangle+1) \ln (\langle N\rangle+1)-\langle N\rangle \ln \langle N\rangle=\ln \langle N\rangle+\langle N\rangle \ln \left(1+\frac{1}{\langle N\rangle}\right) \sim \ln \langle N\rangle$ at $T=0$. This reflects the fact that we are working with GCE where the particle-number fluctuation is still permissible at $T=0$ and the fluctuation amplitude $\delta N \sim\langle N\rangle$. However, as we show below, the mutual information still converges to the correct zero temperature limit, the entanglement entropy, at least to the leading order.

The von Neumann entropy for a subsystem $A$ is given by

$$
E_{A}^{(\mathrm{GCE})}=\left(N_{A}+1\right) \ln \left(N_{A}+1\right)-N_{A} \ln N_{A}=\ln N_{A}+N_{A} \ln \left(1+\frac{1}{N_{A}}\right),
$$

where $N_{A}=\langle n\rangle L_{A}$ is the average total particle number in the subsystem $A$. In the large $N$ limit, the second term converges to 1 . So the mutual information is given by:

$$
E_{M} \equiv \frac{1}{2}\left(E_{A}+E_{B}-S_{G C E T=0}\right)=\frac{1}{2}\left(\ln \frac{N_{A} N_{B}}{N}+1\right) .
$$

For $N_{A} \leq N_{B}$, we have

$$
E_{M} \simeq \frac{1}{2} \ln N_{A}+\mathcal{O}(1)
$$

This agrees with Eq. (11) at the leading order.

\subsection{Mutual information}

According to our Eq. (25) and Eq. (26), to obtain the von Neumann entropy of the reduced density matrix, all what we have to do is to diagonalize the truncated two-point correlation function matrix. Fortunately, within this infinite-range hopping model, this is rather simple. For a finite system, we can obtain a general result for all temperatures:

$$
\begin{aligned}
G_{i j} & =\left\langle\hat{a}_{i}^{\dagger} \hat{a}_{j}\right\rangle=\left\langle\frac{1}{L} \sum_{k} e^{-i k(i-j)} \hat{b}_{k}^{\dagger} \hat{b}_{k}\right\rangle \\
& =\frac{1}{L}\left\langle\hat{b}_{0}^{\dagger} \hat{b}_{0}\right\rangle+\frac{1}{L} \sum_{k \neq 0} e^{-i k(i-j)}\left\langle\hat{b}_{k}^{\dagger} \hat{b}_{k}\right\rangle=\frac{\left\langle N_{0}\right\rangle}{L}+\frac{\left\langle N_{k}\right\rangle}{L} \sum_{k \neq 0} e^{-i k(i-j)} \\
& =\frac{\left\langle N_{0}\right\rangle}{L}+\left(\delta_{i j}-\frac{1}{L}\right)\left\langle N_{k}\right\rangle .
\end{aligned}
$$

In the above calculation, we have made use of the fact that $\left\langle N_{k}\right\rangle$ is $k$-independent. This matrix is easily diagonalized. For a system of size $L$, and a $G$ truncated to a size of $L_{A} \times L_{A}$ denoted by $G_{A}$, the eigenvalues are

$$
g_{1}=\frac{L_{A}\left\langle N_{0}\right\rangle}{L}+\frac{L-L_{A}}{L}\left\langle N_{k}\right\rangle, g_{l}=\left\langle N_{k}\right\rangle \text { for } l=2, \ldots, L_{A} .
$$

Now the von Neumann entropy of subsystem $A$ can be calculated directly from above result:

$$
\begin{aligned}
E_{A} & =\sum_{l=1}^{L_{A}}\left(\left(1+g_{l}\right) \ln \left(1+g_{l}\right)-g_{l} \ln g_{l}\right) \\
& =\left(1+\frac{L_{A}\left\langle N_{0}\right\rangle}{L}+\frac{L-L_{A}}{L}\left\langle N_{k}\right\rangle\right) \ln \left(1+\frac{L_{A}\left\langle N_{0}\right\rangle}{L}+\frac{L-L_{A}}{L}\left\langle N_{k}\right\rangle\right) \\
& -\left(\frac{L_{A}\left\langle N_{0}\right\rangle}{L}+\frac{L-L_{A}}{L}\left\langle N_{k}\right\rangle\right) \ln \left(\frac{L_{A}\left\langle N_{0}\right\rangle}{L}+\frac{L-L_{A}}{L}\left\langle N_{k}\right\rangle\right) \\
& +\left(L_{A}-1\right)\left[\left(1+\left\langle N_{k}\right\rangle\right) \ln \left(1+\left\langle N_{k}\right\rangle\right)-\left\langle N_{k}\right\rangle \ln \left(\left\langle N_{k}\right\rangle\right)\right] .
\end{aligned}
$$


Combining the above with Eq. (19), we can obtain the mutual information for a general bipartite system:

$$
\begin{aligned}
& E_{M}=\frac{1}{2}\left(E_{A}+E_{B}-S\right) \\
& =\frac{1}{2}\left[\left(1+\frac{L_{A}\left\langle N_{0}\right\rangle}{L}+\frac{L_{B}}{L}\left\langle N_{k}\right\rangle\right) \ln \left(1+\frac{L_{A}\left\langle N_{0}\right\rangle}{L}+\frac{L_{B}}{L}\left\langle N_{k}\right\rangle\right)\right. \\
& -\left(\frac{L_{A}\left\langle N_{0}\right\rangle}{L}+\frac{L_{B}}{L}\left\langle N_{k}\right\rangle\right) \ln \left(\frac{L_{A}\left\langle N_{0}\right\rangle}{L}+\frac{L_{B}}{L}\left\langle N_{k}\right\rangle\right) \\
& +\left(1+\frac{L_{B}\left\langle N_{0}\right\rangle}{L}+\frac{L_{A}}{L}\left\langle N_{k}\right\rangle\right) \ln \left(1+\frac{L_{B}\left\langle N_{0}\right\rangle}{L}+\frac{L_{A}}{L}\left\langle N_{k}\right\rangle\right) \\
& -\left(\frac{L_{B}\left\langle N_{0}\right\rangle}{L}+\frac{L_{A}}{L}\left\langle N_{k}\right\rangle\right) \ln \left(\frac{L_{B}\left\langle N_{0}\right\rangle}{L}+\frac{L_{A}}{L}\left\langle N_{k}\right\rangle\right) \\
& \left.-\left(1+\left\langle N_{k}\right\rangle\right) \ln \left(1+\left\langle N_{k}\right\rangle\right)+\left\langle N_{k}\right\rangle \ln \left\langle N_{k}\right\rangle-\left(1+\left\langle N_{0}\right\rangle\right) \ln \left(1+\left\langle N_{0}\right\rangle\right)+\left\langle N_{0}\right\rangle \ln \left\langle N_{0}\right\rangle\right] .
\end{aligned}
$$

Next, we shall discuss the asymptotic behavior of $E_{M}$ in different temperature regions and with different partitions.

(1) $L_{A} \ll L, T>T_{C}$ :

in this case, $\left\langle N_{0}\right\rangle$ and $\left\langle N_{k}\right\rangle \simeq\langle n\rangle$ are both of order one, so $\frac{L_{A}\left\langle N_{0}\right\rangle}{L} \rightarrow 0, \frac{L_{A}\left\langle N_{k}\right\rangle}{L} \rightarrow 0, L_{B} \simeq L$. Thus

$$
E_{M} \simeq \mathcal{O}\left(\frac{L_{A}}{L}\right) .
$$

(2) $L_{A}=L_{B}=\frac{L}{2}, T>T_{C}$ : in this case, $E_{M}$ is reduced to

$$
\begin{aligned}
E_{M} & =\frac{1}{2}\left(\left(2+\left\langle N_{0}\right\rangle+\left\langle N_{k}\right\rangle\right) \ln \left(1+\frac{1}{2}\left(\left\langle N_{0}\right\rangle+\left\langle N_{k}\right\rangle\right)\right)\right. \\
& -\left(\left\langle N_{0}\right\rangle+\left\langle N_{k}\right\rangle\right) \ln \left(\left\langle N_{0}\right\rangle / 2+\left\langle N_{k}\right\rangle / 2\right) \\
& -\left(1+\left\langle N_{k}\right\rangle\right) \ln \left(1+\left\langle N_{k}\right\rangle\right)+\left\langle N_{k}\right\rangle \ln \left\langle N_{k}\right\rangle \\
& \left.-\left(1+\left\langle N_{0}\right\rangle\right) \ln \left(1+\left\langle N_{0}\right\rangle\right)+\left\langle N_{0}\right\rangle \ln \left\langle N_{0}\right\rangle\right)
\end{aligned}
$$

which can also be written as an explicit function of $L,\langle n\rangle$ and $T$ using previous results.

(3) $L_{A} \ll L, T<T_{C}$ : in this case, $\left\langle N_{0}\right\rangle$ becomes a macroscopic number, while $\left\langle N_{k}\right\rangle$ remains to be of order 1. Therefore we isolate the contribution from $\left\langle N_{0}\right\rangle$, and other terms are of order $\mathcal{O}(1)$ :

$$
E_{M}=\frac{1}{2} \ln \left(1+\frac{L_{A}\left\langle N_{0}\right\rangle}{L}\right)+\mathcal{O}(1)
$$

If we define $\left\langle N_{A 0}\right\rangle=\frac{L_{A}\left\langle N_{0}\right\rangle}{L}$ to be the average particle number in the condensate of the subsystem, then

$$
E_{M}=\frac{1}{2} \ln \left\langle N_{A 0}\right\rangle+\mathcal{O}(1)
$$

(4) $L_{A}=L_{B}=\frac{L}{2}, T<T_{C}$ : the leading contribution is again obtained by keeping $\left\langle N_{0}\right\rangle$ 's contribution only:

$$
E_{M}=\frac{1}{2} \ln \left(\frac{1}{4}\left\langle N_{0}\right\rangle+1\right)+\mathcal{O}(1)=\frac{1}{2} \ln \left\langle N_{A 0}\right\rangle+\mathcal{O}(1) .
$$

(5) $L_{A} \ll L, T=T_{C}$ : as we calculated before, $\left\langle N_{0}\right\rangle$ diverges as $\sqrt{L}$. When $L_{A} \ll L$, according to Eqs. (36) and (24), we have

$$
E_{M}=\frac{1}{2} \ln \left(1+\frac{L_{A} \sqrt{(\langle n\rangle+1)\langle n\rangle}}{\sqrt{L}}\right)+\mathcal{O}(1)=\frac{1}{2} \ln \left(\langle n\rangle \frac{L_{A}}{\sqrt{L}}\right)+\mathcal{O}(1)
$$

For such partition, the scaling behavior of mutual information depends on the ratio $\frac{L_{A}}{\sqrt{L}}$. If we consider $L_{A}$ is a small but still finite fraction of $L$, the scaling behavior of the mutual information still persists: $E_{M}=$ $\frac{1}{4} \ln \left(\langle n\rangle L_{A}\right)+\mathcal{O}(1)$. 


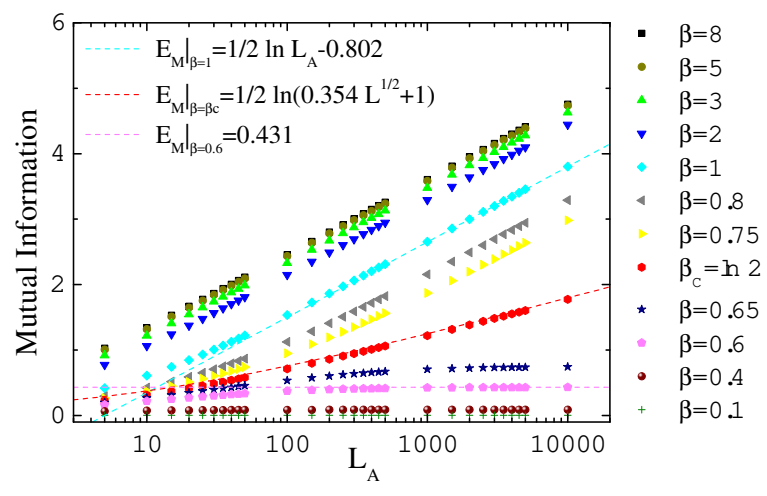

FIG. 1: (Color online) Numerical calculation of mutual information for the infinite-range hopping model for equally partitioned systems with average density $\langle n\rangle=1$. The scatters are numerical data, while the dash lines are obtained from our analytic results corresponding to the particular temperature. We see exactly what our analytic results tell us: above $T_{C}=\frac{1}{\ln 2}$ the mutual information saturates; below $T_{C}, E_{M} \simeq \frac{1}{2} L_{A}$; at $T_{C}, E_{M} \simeq \frac{1}{4} L_{A}$. Note that the analytic results (dash lines) deviate from the numerical calculation because we only keep terms to the subleading order; terms that goes to zero [i.e., of order $\left.\mathcal{O}\left(\frac{1}{L_{A}}\right)\right]$ in the thermodynamic limit are neglected.

(6) $L_{A}=L / 2, T=T_{C}$ : by referring to Eqs. (38) and (24), we have

$$
E_{M}=\frac{1}{2} \ln \left(\frac{1}{4} \sqrt{(\langle n\rangle+1)\langle n\rangle L}\right)+\mathcal{O}(1)=\frac{1}{4} \ln \left(\langle n\rangle L_{A}\right)+\mathcal{O}(1)
$$

To sum up, from this study, we find that the extensive part of the thermal entropy of the whole system is canceled out in the mutual information. Below $T_{C}$ the mutual information is dominated by contribution from the condensate. Even above $T_{C}$, contribution from the condensate is of the same order as that from the excited states. The mutual information really characterizes the quantum feature of the system.

To visualize the behavior of mutual information, we present a numerical calculation for the mutual information of this model in Fig. (1). This is done by numerically diagonalizing the truncated two-point correlation function matrix then computing the von Neumann entropy of the reduced density matrix from those eigenvalues. The system is equally partitioned, $\langle n\rangle=1$, so $T_{C}=\frac{1}{\ln 2}$. We see exactly what our analytic results tell us: above $T_{C}$ the mutual information saturates; below $T_{C}, E_{M} \simeq \frac{1}{2} L_{A}$; at $T_{C}, E_{M} \simeq \frac{1}{4} L_{A}$. Note that in the plot, the analytic results (dash lines) deviate from the numerical calculation because we only keep terms to the subleading order; terms that goes to zero [i.e. of order $\left.\mathcal{O}\left(\frac{1}{L_{A}}\right)\right]$ in the thermodynamic limit are neglected.

\section{NUMERICAL STUDY OF MUTUAL INFORMATION IN ONE-DIMENSION WITH LONG-RANGE HOPPING}

In this section, we shall present our results of numerical study of the mutual information of free bosons living on a one dimensional lattice. In this numerical study, we adopt our previous method, and calculate the von Neumann entropy of the reduced density matrix from the eigenvalues of the truncated two-point correlation function matrix. Throughout this calculation, we hold the average density fixed at $\langle n\rangle=1$ (which means we keep adjusting the chemical potential at different temperatures) and consider equal partition only.

It is well known that for nearest-neighbor (NN) (or other short-range) hopping models whose dispersion relation at long-wave length takes the form $\epsilon(k) \sim k^{2}$, a finite temperature BEC can only exist in three dimensions (3D). However, 3D is in general very challenging for a numerical study that requires large system sizes. Moreover, in 3D the mutual information is dominated by area law [10], which renders the logarithmic divergence suggested by our study in Sec. III sub-leading and thus difficult to isolate. For both of these reasons, it is desirable to study a model in 1D with BEC at finite $T$. In 1D, the short-range hopping model does not support BEC at finite temperature. To stabilize a condensate in 1D, we introduce power-law long-range hopping in our free boson model to modify its long-wave length dispersion. This is similar to what was done in Ref. [33], in which the authors introduced long-range interaction between spins to stabilize magnetic order in 1D. The Hamiltonian with long-range hopping is obtained by setting $t_{i j}$ 
in Eq. (1) to the following form, tuned by a parameter $\gamma$ :

$$
H=-\sum_{i j} \frac{t}{|i-j|^{\gamma}} a_{i}^{\dagger} a_{j}=-2 t \sum_{k}\left(\sum_{n=1}^{L-1} \frac{\cos (n k)}{n^{\gamma}}\right) b_{k}^{\dagger} b_{k}=\sum_{k} \varepsilon_{\gamma}(k) b_{k}^{\dagger} b_{k} .
$$

We will show that the long wave-length dispersion is modified to be $\varepsilon_{\gamma}(k) \sim k^{\gamma-1}$ for $\gamma<3$, as a result if which a finite temperature BEC exists for $\gamma<2$.

Consider the eigenenergy function $\varepsilon_{\gamma}(k)=-2 t \sum_{n=1}^{L-1} \frac{\cos (n k)}{n^{\gamma}}$ in the thermodynamic limit:

$$
\varepsilon_{\gamma}(k)=-2 t \sum_{n=1}^{\infty} \frac{\cos (n k)}{n^{\gamma}}=-2 t \operatorname{Re}\left[\sum_{n} \frac{e^{i n k}}{n^{\gamma}}\right]=-2 t \operatorname{Re}[F(\gamma, i k)],
$$

where $F(\gamma, v)$ is the Bose-Einstein integral function [34] defined as:

$$
F(\gamma, v)=\frac{1}{\Gamma(\gamma)} \int d x \frac{x^{\gamma-1}}{e^{x+v}-1}=\sum_{n=1}^{\infty} \frac{e^{-n v}}{n^{\gamma}}
$$

The analytic properties of $F(\gamma, v)$ near $v=0$ are known [34]:

$$
F(\gamma, v)= \begin{cases}\Gamma(1-\gamma) v^{\gamma-1}+\sum_{n=0}^{\infty} \frac{\zeta(\gamma-n)}{n !}(-v)^{n}, & \gamma \notin \mathbb{Z} \\ \frac{(-v)^{\gamma-1}}{(\gamma-1) !}\left[\sum_{r=1}^{\gamma-1} \frac{1}{r}-\ln (v)\right]+\sum_{n \neq \gamma-1}^{\infty} \frac{\zeta(\gamma-n)}{n !}(-v)^{n}, & \gamma \in \mathbb{Z}\end{cases}
$$

where $\zeta(x)$ is the Riemann zeta function. Thus we find that $\varepsilon_{\gamma}(k) \rightarrow k^{\gamma-1}$ for small $k$ when $1<\gamma<3$. When $\gamma>3$, the low energy dispersion is dominated by the $k^{2}$ term. When $\gamma \leq 1, \varepsilon_{\gamma}(k)$ is not well-defined in the thermodynamic limit; in order to have a well-defined thermodynamic limit, the hopping energy $t$ must be properly scaled by the system size in this case.

Next we will consider the thermodynamics of this model with different $\gamma$ and demonstrate that for $\gamma<2$, we indeed have a finite temperature BEC. At low temperature, only the small $k$ part of the spectrum is important. For $1<\gamma<3$ we consider free bosons with a dispersion $\sigma k^{\gamma-1}$. Here $\sigma=-2 t \Gamma(1-\gamma)$ is given in Eq. (44). The average density of such system in the thermodynamic limit is given by:

$$
\frac{\langle N\rangle}{L}=\frac{1}{2 \pi} \int d k\left\langle N_{k}\right\rangle=\frac{1}{2 \pi} \frac{\sigma^{\frac{1}{1-\gamma}}}{(\gamma-1)} \int_{0}^{\infty} d \varepsilon \frac{\varepsilon^{\frac{1}{\gamma-1}-1}}{z^{-1} e^{\beta \varepsilon}-1}=\frac{(\beta \sigma)^{\frac{1}{1-\gamma}}}{2 \pi(\gamma-1)} \Gamma\left(\frac{1}{\gamma-1}\right) g_{\frac{1}{\gamma-1}}(z),
$$

where $z=e^{\beta \mu}, \beta=\frac{1}{T}$ is the inverse temperature, and $g_{v}(z)=\frac{1}{\Gamma(v)} \int_{0}^{\infty} d x \frac{x^{v-1}}{z^{-1} e^{x}-1}$ is the Bose-Einstein integral function. To have a finite temperature BEC, $\langle N\rangle / L=\langle n\rangle$ must remain finite when $z \rightarrow 1$, which indicates $\left.\frac{1}{\gamma-1}\right\rangle 1$, because for $v \leq 1, g_{v}(1)$ diverges.

To have a better understanding of the thermodynamics of this model, in Fig. (2) we present a numerical calculation of $T_{C}$. The exact average density of the system is:

$$
\frac{\langle N\rangle}{L}=\frac{1}{2 \pi} \int_{-\pi}^{\pi} d k \frac{1}{e^{\beta\left(\varepsilon_{\gamma}(k)-\mu\right)}-1}
$$

where $\varepsilon_{\gamma}(k)$ is the eigenenergy function given in Eq. (42). $T_{C}$ is computed by setting $\mu=\varepsilon_{\gamma}(0)$ and then solving this equation numerically. According to Fig. $2, T_{C}$ grows monotonically from 0 to $\infty$ as $\gamma$ goes from 2 to 1 . The divergent behavior of $T_{C}$ as $\gamma \rightarrow 1$ is a consequence of the divergent bandwidth in that limit.

According to our study of the infinite range hopping model, above $T_{c}$ the mutual information should saturate as the system size grows. Below $T_{C}$, the mutual information has a scaling behavior $E_{M} \simeq \frac{1}{2} \ln L_{A}$; for $T=T_{C} E_{M} \simeq \frac{1}{4} \ln L_{A}$ for equally partitioned system in that model. We expect the $\ln L_{A}$ scaling behavior both below $T_{C}$ and at $T_{C}$ to persist in the long-range hopping model. As we shall see later, this is indeed the case. However, the details of the scaling behavior (i.e. the prefactor) can be different for different $\gamma$. To study this scaling behavior, we fix the temperature and examine the mutual information as a function of system size. This is desirable because, if our conjecture according to the study of infinite-range hopping model is correct, the mutual information will be proportional to ln $L_{A}$ when $T \leq T_{C}$. 


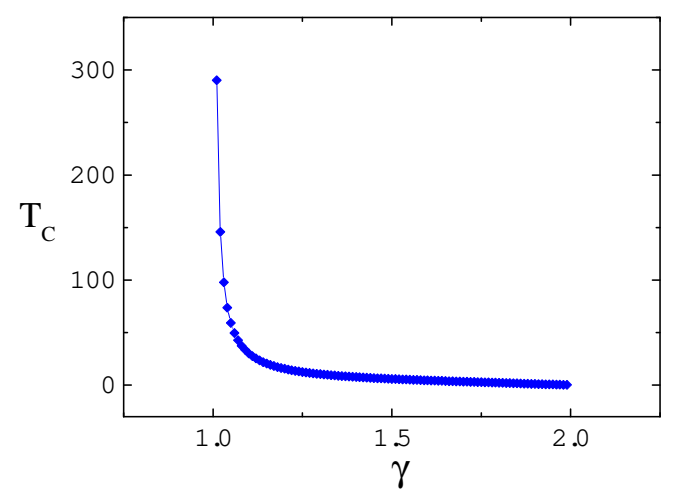

FIG. 2: (Color online) Numerical calculation of $T_{C}$ (line + symbols) for the long-range hopping model in the thermodynamic limit. $T_{C}$ is measured in unit of the hopping energy $t$ which is set to 1 . As one can see, $T_{C}$ grows monotonically from 0 to $\infty$ as $\gamma$ goes from 2 to 1 . The divergent behavior of $T_{C}$ as $\gamma \rightarrow 1$ is a consequence of the divergent bandwidth.

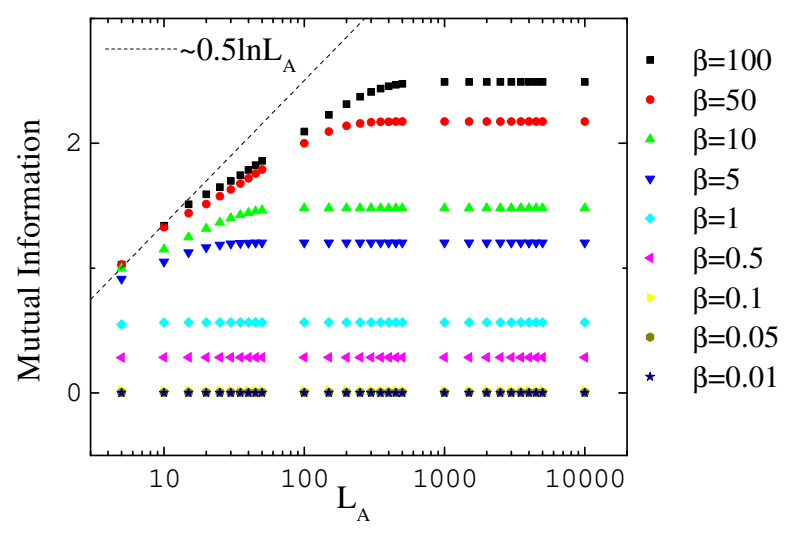

FIG. 3: (Color online) Mutual information of the nearest neighbor hopping model plotted against subsystem size on a logarithmic scale. Average density is set to $\langle n\rangle=1$, and the system is equally partitioned, $L=2 L_{A}$. The black dash line is $E_{M} \sim \frac{1}{2} \ln L_{A}$. This line will be in other graphs for comparison as well. Clearly, the mutual information saturates when the system size grows large enough.

Before we demonstrate our results in the long-range hopping model, first let us verify our analysis in the NN hopping model in which no BEC would occur. This case actually corresponds to $\gamma \rightarrow \infty$. The Hamiltonian is given by restricting the hopping in Eq. (1) to the nearest neighbors only:

$$
H_{n . n}=-t \sum_{\langle i j\rangle} \hat{c}_{i}^{\dagger} \hat{c}_{j} .
$$

Figure 3 is a linear-log plot of mutual information against subsystem size at different temperatures. Throughout our study we shall consider equal partition only, i.e., $L_{A}=L / 2$. The average density is also set to $\langle n\rangle=1$ here, so are the other results we will show later. Clearly, at a fixed temperature, the mutual information saturates as the system size grows. At low temperatures, small systems can be considered in the zero temperature limit. This leads to the mutual information growing as $\sim \frac{1}{2} \ln L_{A}$, until saturation kicks in.

Next we consider $1<\gamma<2$. Now the finite temperature transition emerges, and the signature for the transition in mutual information - the logarithmic scaling with (sub-)system size also emerges. In Fig. 4, we plot the mutual information (scatters) for $\gamma=1.7$ at different temperatures. The best fit (cyan dash line corresponding to the diamond data points) for $\beta=0.5>\beta_{C}$ gives $E_{M}=0.2405 \ln L_{A}+0.214$. At $\beta_{C}=0.297$, the scaling behavior is fit (magenta dash line) as $E_{M}=0.1226 \ln L_{A}+0.1688$. Both behaviors agrees qualitatively with what has been suggested by our analytic study of the infinite-range hopping model. When the temperature is well below $T_{C}$, we have the logarithmic 


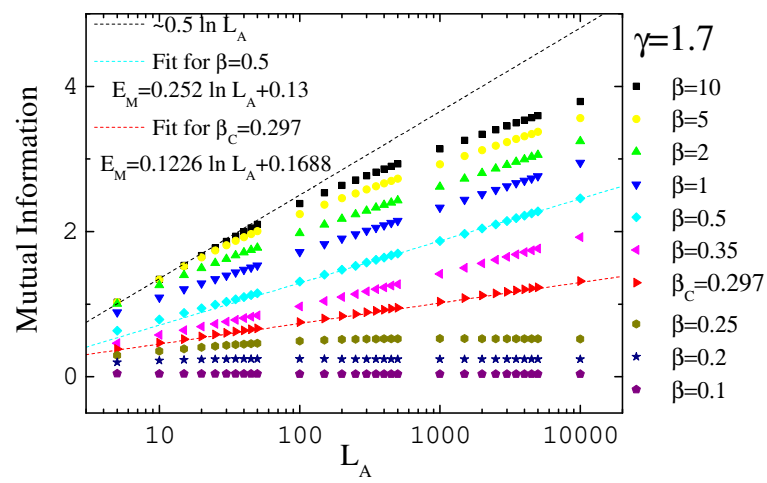

FIG. 4: (Color online) Mutual information of the long-range hopping model with the parameter $\gamma=1.7$ as a function of subsystem size on a logarithmic scale, at various (inverse) temperatures. The average boson density $\langle n\rangle$ is set to 1 , and the system is equally partitioned, $L_{A}=L / 2$. The scaling behavior for inverse temperature $\beta=0.5$ goes as $E_{M}=0.2405 \ln L_{A}+0.214$ (cyan dash line corresponding to the diamond data points). At the transition point, $\beta=\beta_{C}=0.297$, the scaling law is fit to be $E_{M}=0.1226 \ln L_{A}+0.1688$ (red dash line corresponding to the right triangle data points).

scaling behavior: a set of parallel linear lines on this logarithm scale plot for different temperatures. However, the prefactor is significantly different from that of the infinite range hopping model which is $\frac{1}{2}$. In fact, by calculating mutual information of different $\gamma$ 's, we find that the prefactor varies as $\gamma$ changes. For very low temperature, small systems again effectively fall into the zero temperature region, and the mutual information restores to the $\sim \frac{1}{2} \ln L_{A}$ behavior as in zero temperature. But when the system size becomes large it crosses back to the finite temperature scaling behavior again. This is evident for $\beta>1$ in the figure. For temperature close to but still below $T_{C}$, small systems behave differently: the mutual information scales more like the line for $\beta=\beta_{C}$, and it bends up as the system size increases and finally crosses back to its genuine behavior below $T_{C}$. For temperature close to $T_{C}$ but now above, small systems behave the other way: the mutual information bends downwards and saturates at large system size. $\beta_{c}$ serves as a very distinctive boundary between the above two different bending behaviors. The latter two bending features are also present in Fig. 1, our numerical verification of the infinite-range hopping model. But the first feature at very low temperature is missing in Fig. 1 since in that case the mutual information scales the same way as the entanglement entropy.

Very similar behaviors were observed for the entire range $1<\gamma<2$. Representative results are presented in Figs. 5 and 6 for $\gamma=1.5$ and 1.3 respectively. We thus conclude that for the entire range $1<\gamma<2$, mutual information saturates for $T>T_{C}$, while it diverges logarithmically with increasing subsystems size, for both $T<T_{C}$ and $T=T_{C}$. The coefficients in front of the logarithms are $\gamma$-dependent.

\section{SUMMARY AND CONCLUDING REMARKS}

In this paper we have studied entanglement properties of free non-relativistic Bose gases. At zero temperature, all particles fall into the ground state, and we find the entanglement entropy diverges as the logarithm of the particle number in the subsystem. At finite temperatures, we studied the natural generalization of entanglement entropy the mutual information. We find the mutual information has a similar divergence in the presence of a Bose-Einstein condensate. When the system is above $T_{C}$ or does not have a condensate, the mutual information saturates for large subsystem size. It should be noted that for the special models we studied in this paper there is no area-law contribution to the mutual information, thus the contribution from the condensate, when present, dominates the mutual information. In more generic models in two- or three-dimensions where an area-law contribution is present, we expect such logarithmic divergent contribution from the condensate to be present as a sub-leading term in the subsystem-size dependence of the entanglement entropy and mutual information.

Physically it is easy to understand why the condensate makes such an important contribution to entanglement. First of all, BEC is intrinsically a quantum process, just like entanglement reflects the intrinsically quantum nature of the system. More specifically, when a (macroscopically) large number of particles occupy the same state (at $k=0$ ), they are necessarily delocalized throughout the sample, giving rise to entanglement between blocks.

Just like in our previous work on a very different system [27], our results here suggest that conventional ordering, 


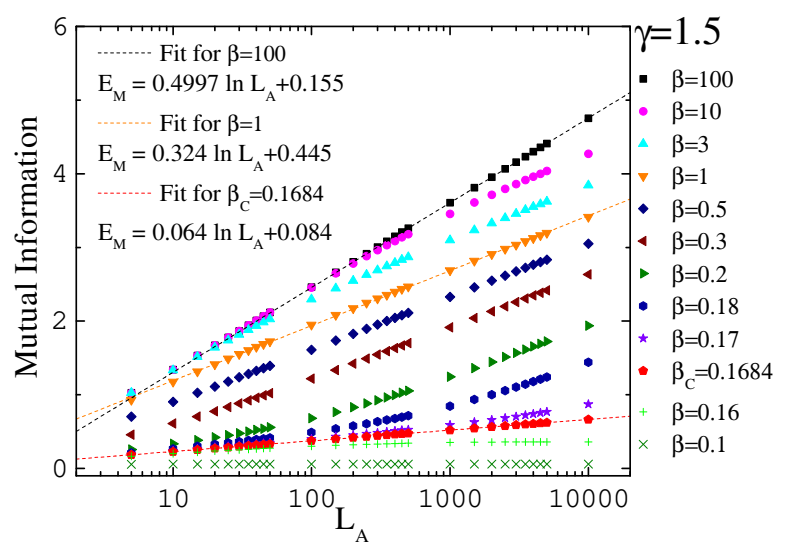

FIG. 5: (Color online) Mutual information of the long-range hopping model with the parameter $\gamma=1.5$ as a function of subsystem size on a logarithmic scale, at various (inverse) temperatures. The average boson density $\langle n\rangle$ is set to 1 , and the system is equally partitioned, $L_{A}=L / 2$. The mutual information for $\beta=1$ is fit to scale as $E_{M} \simeq 0.324 \ln L+0.445$ (orange dash line). At $\beta_{C}=0.16843$, we observer a weaker scaling behavior which is fit to be $E_{M}=0.064 \ln L_{A}+0.084$ (red dash line).

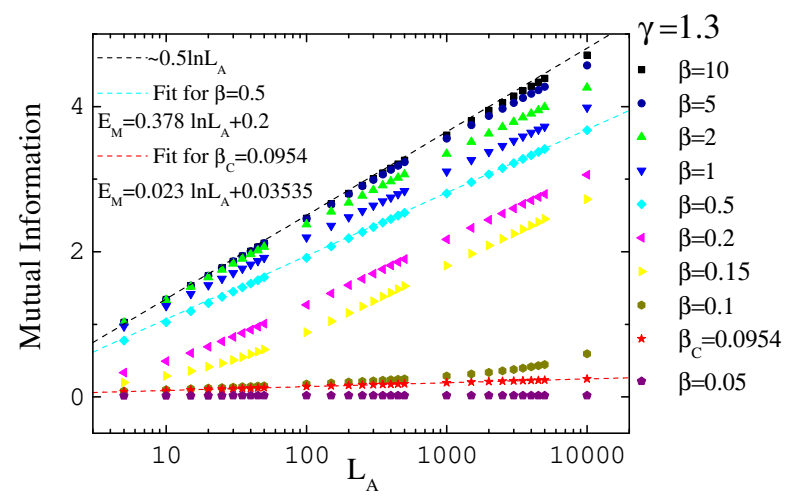

FIG. 6: (Color online) Mutual information of the long-range hopping model with the parameter $\gamma=1.3$ as a function of subsystem size on a logarithmic scale, at various (inverse) temperatures. The average boson density $\langle n\rangle$ is set to 1 , and the system is equally partitioned, $L_{A}=L / 2$. The best fitting for $\beta=0.5>\beta_{C}$ (cyan dash line) gives $E_{M}=0.378 \ln L_{A}+0.2$. At $\beta_{C}=0.0954$, the scaling behavior is fit (red dash line) as $E_{M}=0.023 \ln L_{A}+0.03535$.

like BEC, makes a logarithmic contribution to entanglement. One thus needs to take caution when using entanglement as a diagnostic for exotic phases (such as topological phases) or quantum criticality.

\section{ACKNOWLEDGMENTS}

We thank Dr. Libby Heaney for a useful correspondence. This work was supported by NSF Grant No. DMR0704133. The authors thank the Kavli Institute for Theoretical Physics (KITP) for the warm hospitality during the completion of this work. The work at KITP was supported in part by National Science Foundation Grant No. PHY-0551164.

[1] L. Amico, R. Fazio, A. Osterloh, and V. Vedral, Rev. Mod. Phys. 80, 517 (2008).

[2] C. Holzhey, F. Larsen, and F. Wilczek, Nucl. Phys. B 424, 443 (1994). 
[3] P. Calabrese and J. Cardy, J. Stat. Mech: Theory Exp. 0406, 002 (2004).

[4] H. Casini and M. Huerta, J. Stat. Mech: Theory Exp. 0512, 012 (2005).

[5] G. Adesso and F. Illuminati, J. Phys. A 40, 7821 (2007).

[6] S. L. Braunstein and P. van Loock, Rev. Mod. Phys. 77, 513 (2005).

[7] S. Sachdev, Quantum Phase Transition (Cambridge University Press, 1999).

[8] X.-G. Wen, Int. J. Mod. Phys. B 4, 239 (1990).

[9] L. Bombelli, R. K. Koul, J. Lee, and R. D. Sorkin, Phys. Rev. D 34, 373 (1986).

[10] M. Srednicki, Phys. Rev. Lett. 71, 666 (1993).

[11] J. Eisert, M. Cramer, and M. B. Plenio arXiv: 0808.3773 (2008).

[12] M. M. Wolf, Phys. Rev. Lett. 96, 010404 (2006).

[13] D. Gioev and I. Klich, Phys. Rev. Lett. 96, 100503 (2006).

[14] T. Barthel, M.-C. Chung, and U. Schollwock, Phys. Rev. A 74, 022329 (2006).

[15] W.F. Li, L.T. Ding, R. Yu, T. Roscilde, and S. Haas, Phys. Rev. B 74, 073103 (2006).

[16] G. Vidal, J. I. Latorre, E. Rico, and A. Kitaev, Phys. Rev. Lett. 90, 227902 (2003).

[17] G. Refael and J. E. Moore, Phys. Rev. Lett. 93, 260602 (2004).

[18] R. Santachiara, J. Stat. Mech. L06002 (2006).

[19] A. Feiguin, S. Trebst, A. W. W. Ludwig, M. Troyer, A. Kitaev, Z. Wang, and M. H. Freedman, Phys. Rev. Lett. 98, 160409 (2007).

[20] N. E. Bonesteel and K. Yang, Phys. Rev. Lett. 99, 140405 (2007).

[21] M. Levin and X.-G. Wen, Phys. Rev. Lett. 96, 110405 (2006).

[22] A. Kitaev and J. Preskill, Phys. Rev. Lett. 96, 110404 (2006).

[23] E. Fradkin and J. E. Moore, Phys. Rev. Lett. 97, 050404 (2006).

[24] J. I. Latorre, R. Orús, E. Rico, and J. Vidal, Phys. Rev. A 71, 064101 (2005).

[25] T. Barthel, S. Dusuel, and J. Vidal, Phys. Rev. Lett. 97, 220402 (2006).

[26] J. Vidal, S. Dusuel, and T. Barthel, J. Stat. Mech.: Theory Exp. 2007, P01015 (2007).

[27] W. Ding, N. E. Bonesteel, and K. Yang, Phys. Rev. A 77, 052109 (2008).

[28] M. Cramer, J. Eisert, M. B. Plenio, and J. D. Dreißig, Phys. Rev. A 73, 012309 (2006).

[29] M. M. Wolf, F. Verstraete, M. B. Hastings, and J. I. Cirac, Phys. Rev. Lett. 100, 070502 (2008)

[30] A similar calculation for a BEC condensate of bosons who share a particular Gross-Pitaevskii wave function has been done by Klich, Refael, and Silva [I. Klich, G. Refael, and A. Silva Phys. Rev. A, 74, 032306 (2006)]; the scaling behavior of the entanglement entropy was pointed out earlier by Simon [C. Simon, Phys. Rev. A 66, 052323 (2002)].

[31] It is one half of the "mutual information" introduced in Refs. [28] and [29], and reduces to the entanglement entropy when $\rho$ is that of a pure state. The same definition was used by Castelnovo and Chamon [Claudio Castelnovo, and Claudio Chamon, Phys. Rev. B 76, 174416 (2007)]; the name "mutual information" may be first coined by Adami and Cerf [C. Adami and N.J. Cerf, Phys. Rev. A 56, 3470 (1997)] and Vedral, Plenio, Rippin and Knight [V. Vedral, M.B. Plenio, M.A. Rippin, and P.L. Knight, Phys. Rev. Lett. 78, 2275 (1997)], although Stratonovich [R. L. Stratonovich, Izv. Vyssh. Uchebn. Zaved., Radiofiz. 8, 116 (1965); Probl. Inf. Transm. 2, 35 (1966)] considered this quantity already in the mid-1960s.

[32] I. Peschel, J. Phys. A: Math. Gen. 36, L205 (2003)

[33] E. Yusuf, A. Joshi, and K. Yang, Phys. Rev. B 69, 144412 (2004).

[34] J. E. Robinson, Phys. Rev. 83, 678 (1951). 